\title{
Public Health Law
}

Office for State, Tribal, Local and Territorial Support

Centers for Disease Control and Prevention

\section{Prescription Drug Time and Dosage Limit Laws}

The United States is in the midst of an unprecedented epidemic of prescription drug overdose deaths. ${ }^{1}$ Nearly 44,000 people died of drug overdoses in 2013 , and just over half of these deaths $(22,767)$ were caused by overdoses involving prescription drugs. ${ }^{2}$ The vast majority of prescription drug overdose deaths in $2013(16,235)$ involved a prescription opioid pain reliever (OPR), which is a drug derived from the opium poppy or synthetic versions of it such as oxycodone, hydrocodone, or methadone. ${ }^{3}$ The prescription drug overdose epidemic has not affected all states equally, thus overdose death rates vary widely among states.

States have the primary responsibility to regulate and enforce prescription drug practice. Although state laws are commonly used to prevent injuries and their benefits have been demonstrated for a variety of injury types, ${ }^{4}$ there is little information on the effectiveness of state statutes and regulations designed to prevent prescription drug abuse and diversion. This menu is a first step in assessing laws on prescription drug time and dosage limits by creating an inventory of state legal strategies in this domain.

\section{Introduction}

To combat the prescription drug overdose epidemic, many states have enacted laws that set time or dosage limits on the prescribing or dispensing of controlled substances.

\footnotetext{
${ }^{1}$ For the purpose of this document, "overdose death" refers to death resulting from either intentional or accidental overdose, which could be caused by a patient being given the wrong drug, taking the wrong drug in error, or taking too much of a drug inadvertently.

${ }^{2}$ Centers for Disease Control and Prevention, Wide-ranging Online Data for Epidemiologic Research (WONDER) Database (2013) http://wonder.cdc.gov.

${ }^{3}$ Id.

${ }^{4}$ Julie Gilchrist, Richard A. Schieber \& David A. Sleet, Legislative and Regulatory Strategies to Reduce Childhood Injuries, 10 UNINTENTIONAL INJURIES IN CHILDHOOD 111, 111-30 (2000).
} 
A law is included in this resource if it limits prescription drug quantity by time (hours' or days' supply) or amount of drug (dosage units or number of pills). ${ }^{5}$ Time or dosage limit laws concerning benefit plans such as general assistance programs for the elderly (Medicare) or Medicaid programs were included. ${ }^{6}$

Time or dosage limit laws particular to certain facilities, (e.g., long-term care facilities, communitybased care facilities, hospices, home healthcare facilities, nursing facilities, hospitals, and emergency departments) were excluded from this resource. ${ }^{7}$ Laws relating specifically to certain kinds of patients, (e.g., outpatients, discharged patients, and in-patients) were also excluded. Laws regarding payments of claims or reimbursement restrictions of benefit plans are not direct prescription drug limits and were therefore excluded from this resource. ${ }^{8}$ Laws pertaining to treatment of narcotic addiction, drug samples, maintenance drugs, emergency drug kits, multiple-drug single-dosing containers, and collaborative drug therapy management with authorized pharmacists were also excluded.

Laws requiring time or dosage limits were included for physicians (doctors of medicine and doctors of osteopathic medicine) and pharmacists but not for other professions. Forty-seven states ${ }^{9}$ and the District of Columbia have laws that set time or dosage limits for controlled substances.

\section{Time Limitations}

One main category of prescription drug limit laws sets forth time limits (hours' or days' supply) to the supply of prescription drugs. These time limit laws can be further classified by their applicability to certain drugs, certain populations or certain situations.

\section{Time Limits Applicable to Certain Drugs or Types of Prescriptions}

A few states ${ }^{10}$ have laws setting time limits for all prescriptions or all controlled substances. For example, Florida law states

Pharmacists may order the medicinal drug products set forth in each rule subject to the following terms and limitations: ... (3) In any case of dispensing hereunder, the amount or

\footnotetext{
${ }^{5}$ In this menu, the first effective dates of the specific provisions referenced are cited as "[legal citation] (eff. [year])." Where dates were either not provided within the laws or were unclear due to multiple revisions, this fact is cited as "[legal citation] (eff. date unclear, [estimated year])."

${ }^{6}$ Laws related to specific assistance programs like mental health programs, state employees programs, or workers compensation programs were excluded.

${ }^{7}$ Laws related to pain management clinics were included in the scope of research.

${ }^{8}$ See, e.g., 016-06-22 ARK. CODE R. § 213.100 (eff. 2005) (direct prescription drug limit); but see, e.g., 1 TEX. AdMIN. CODE $\S 354.1877$ (eff. 2012) (indirect prescription drug limit).

${ }_{9}^{9}$ Alabama, Arizona, Arkansas, California, Colorado, Connecticut, Florida, Georgia, Hawaii, Idaho, Illinois, lowa, Kansas, Kentucky, Louisiana, Kansas, Kentucky, Louisiana, Maine, Maryland, Massachusetts, Michigan, Minnesota, Mississippi, Missouri, Montana, Nebraska, Nevada, New Hampshire, New Jersey, New Mexico, New York, North Carolina, North Dakota, Ohio, Oklahoma, Oregon, Pennsylvania, Rhode Island, South Carolina, Tennessee, Texas, Utah, Vermont, Virginia, Washington, West Virginia, Wisconsin, and Wyoming.

${ }^{10}$ Five states. See, e.g., FLA. AdMIN. CODE r. 64B15-18.002 (eff. 1986); LA. AdMIN. CODE tit. 46, pt. XLV, § 6506 (eff. 2008); Mo. ANN. STAT. $\S 195.080$ (eff. 1987); N.Y. EdUC. LAW $\S 6807$ (McKinney eff. date unclear); S.C. CODE $\S 44-53-$ 360 (eff. date unclear).
} 
quantity of drug dispensed shall not exceed a [thirty-four]-day supply or standard course of treatment unless subject to the specific limitations in this rule. ${ }^{11}$

Twenty-three states ${ }^{12}$ and the District of Columbia ${ }^{13}$ have laws setting time limits for specific schedules of prescription drugs. For example, Missouri statute provides for day supply limits:

The quantity of Schedule II controlled substances prescribed or dispensed at any one time shall be limited to a thirty-day supply. The quantity of Schedule III, IV or V controlled substances prescribed or dispensed at any one time shall be limited to a ninety-day supply .... The supply limitations provided in this subsection may be increased up to three months if the physician describes on the prescription form or indicates via telephone, fax, or electronic communication to the pharmacy to be entered on or attached to the prescription form the medical reason for requiring the larger supply. The supply limitations provided in this subsection shall not apply if the prescription is dispensed directly to a member of the United States armed forces serving outside the United States. ${ }^{14}$

South Carolina has day supply limits for specific scheduled controlled substances. Its law requires that "[p]rescriptions for controlled substances listed in Schedules III, IV, and V shall not be issued for more than a thirty-one day supply of the substance."15

California law provides for hour supply limits for Schedule II drugs dispensed by the prescriber. Its law states

A practitioner ... may dispense directly to an ultimate user a controlled substance classified in Schedule II in an amount not to exceed a [seventy-two]-hour supply for the patient in accordance with directions for use given by the dispensing practitioner only where the patient is not expected to require any additional amount of the controlled substance beyond the [seventytwo] hours. ${ }^{16}$

\footnotetext{
${ }^{11}$ Fla. AdMIN. CODE r. 64B15-18.002 (eff. 1986). unclear).

${ }^{13}$ D.C. Code Mun. Regs. tit. 22-B, § 1306 (eff. 2006).

${ }^{14}$ MO. ANN. STAT. $\S 195.080$ (eff. 1987).

${ }^{15}$ S.C. CODE ANN. REGS. 61-4.514.1 (eff. date unclear).

${ }^{16}$ CAL. HeAlth \& SAfETy CODE $§ 11158$ (eff. date unclear, 1976-1980).
}

12 See, e.g., CAL.HEALth \& SAFETy CODE $\S 11158$ (eff. date unclear, 1976-1980); Colo. ReV. StAT. ANN. § 18-18-414 (eff. 1992); 24 Del. Admin. Code $§ 4.0$ (eff. 2009); Fla. Stat. AnN. § 893.04 (eff. 2009); IdAho Admin. Code r. 27.01.01.113 (eff. 2012); 720 Ill. COMP. StAT. 570/312 (eff. date unclear); IOWA AdMIn. CodE r. 657-10.25(124) (eff. 2009 ); Ky. REV. STAT. § 218A.205 (eff. 2012); LA. AdMIN. CODE. tit. 46, pt. LIII, § 2745 (eff. 2008); 105 MASS. CODE REGS 700.011 (eff. 1998); 30-20-3001 MisS. Code R. § XIX (eff. 2012); Mo. ANN. StAT. § 195.080 (eff. 1987); NeV. AdMIN. CODE § 453.450 (eff. 2007); N.H. Rev. StAT. § 318-B:9 (eff. 1983); N.J. StAT. ANN. § 45:9-22.19 (eff. 2010); N.M. Code R. § 16.19 .20 (eff. 2002); N.Y. COMP. COdES R. \& Regs. tit. 10, § 80.69 (eff. date unclear); R.I. GEN. LAWS ANN. § 21-28-3.18 (eff. 2006); S.C. Code AnN. Regs. 61-4.514.1 (eff. date unclear); TeX. HEALth \& SAFETy Code ANN.§ 481.074 (eff. 2001); UTAH AdMIN. Code r. 156-37 (eff. date unclear); 20-4-1400 VT. CODE R. § 9.16 (eff. 2009); W. VA. CODE R. § 15-2-7 (eff. date 
Several states ${ }^{17}$ and the District of Columbia ${ }^{18}$ have day or hour limits for oral prescriptions, i.e., those delivered verbally to the pharmacist. For example, a Mississippi regulation states

When a Schedule II controlled substance is needed in a situation in which a written prescription cannot reasonably be obtained it may be considered an emergency situation and a pharmacist may dispense a Schedule II controlled substance pursuant to an oral prescription of a practitioner. A Schedule II controlled substance prescription given in this manner shall be reduced to writing by the pharmacist and shall be for a quantity of medication sufficient for the emergency period, not to exceed [forty-eight] hours. ${ }^{19}$

Eleven states ${ }^{20}$ and the District of Columbia ${ }^{21}$ also have time limits on multiple prescriptions. lowa law provides that "[a]n individual prescriber may issue multiple prescriptions authorizing the patient to receive a total of up to a [ninety]-day supply of a Schedule II controlled substance pursuant to the provisions and limitations of this rule." ${ }^{22}$ Similarly, a New Jersey statute sets day supply limits for multiple prescriptions as long as certain conditions are met. The statute reads

A physician may issue multiple prescriptions authorizing the patient to receive a total of up to a [ninety]-day supply of a Schedule II controlled dangerous substance, provided that the following conditions are met: (1) each separate prescription is issued for a legitimate medical purpose by the physician acting in the usual course of professional practice; [and] (2) the physician provides written instructions on each prescription, other than the first prescription if it is to be filled immediately, indicating the earliest date on which a pharmacy may fill each prescription, [among other conditions] ... ${ }^{23}$

Utah statute contains similar language, stating

A practitioner may issue more than one prescription at the same time for the same Schedule II controlled substance, but only under the following conditions: (A) no more than three prescriptions for the same Schedule II controlled substance may be issued at the same time; [and] (B) no one prescription may exceed a [thirty]-day supply, [among other conditions] . . . ${ }^{24}$

\footnotetext{
${ }^{17}$ Seven states. See, e.g., Colo. REV. StAT. ANN. § 18-18-414 (eff. 1992); FLA. STAT. ANN. § 893.04 (eff. 2009); 30-203001 MISS. CODE R. § XIX (eff. 2012); N.J. ADMIN. CODE § 13:39-7.4 (eff. date unclear, 2005 or 2010); N.M. CODE R. $\S 16.19 .20$ (eff. 2002); N.Y. COMP. COdes R. \& ReGs. tit. 10, § 80.68 (eff. date unclear); UTAH AdMIN. CODE r. 156-37 (eff. date unclear).

${ }^{18}$ D.C. CODE Mun. Regs. tit. 22-B, § 1306 (eff. 2006).

${ }^{19}$ 30-20-3001 MISS. CODE R. § XIX (eff. 2012).

${ }^{20}$ See, e.g., IDAHO ADMIN. CODE r. 27.01.01.113 (eff. 2012); 720 ILL. COMP. STAT. 570/312 (eff. date unclear); IoWA AdMin. Code r. 657-10.25(124) (eff. 2009); LA. AdMIn. Code tit. 46, pt. LIII, § 2745 (eff. 2008); Nev. AdMIN. CODE $\S 453.450$ (eff. 2007); N.J. STAT. ANN. § 45:9-22.19 (eff. 1975); R.I. GEN. LAWS ANN. § 21-28-3.18 (eff. 2006); TEX. HEALTH \& SAFETY CODE ANN. § 481.074 (eff. 2001); UTAH CODE ANN. § 58-37-6 (eff. date unclear, 1971 or 1991); 20-41400 VT. CODE R. $§ 9.16$ (eff. 2009); W. VA. CODE R. § 15-2-7 (eff. date unclear).

${ }^{21}$ D.C. CODE MUN. REGS. tit. 22-B, § 1306 (eff. 2006).

22 IOWA ADMIN. CODE r. 657-10.25(124) (eff. 2009).

${ }^{23}$ N.J. STAT. ANN. § 45:9-22.19 (eff. 1975).

${ }^{24}$ UTAH CODE ANN. § 58-37-6 (eff. date unclear, 1971 or 1991).
} 


\section{Time Limits Applicable to Certain Benefit Plans}

Thirty-six states ${ }^{25}$ and the District of Columbia ${ }^{26}$ provide day or hour supply limits specifically for members of certain benefit plans such as Medicare or Medicaid programs. For example, the Oklahoma law states that under Oklahoma Health Care Authority assistance, "[p]rescription quantities are to be limited to a [thirty-four] day supply except in [some] situations." 27

Similarly, Illinois law provides that under the Pharmaceutical Assistance Program

An authorized pharmacy may not provide [dispense] a beneficiary with more than a [thirty-four]day supply of any covered prescription drug in filling, refilling, or renewing a prescription, except as otherwise specified for medical or utilization control reasons . . ${ }^{28}$

Wyoming law lists supply limits for drugs that may be dispensed to its Medicaid program members. The law includes limits for "[a]Il other drugs. The maximum quantity dispensed for all other conditions shall be a one-month supply" ${ }^{29}$ under its Medicaid program.

\section{Time Limits Applicable to Certain Situations}

Many state prescription drug limit laws set time limits applicable to certain situations.

\section{Emergency-Basis Dispensing}

Twenty-eight states $^{30}$ and the District of Columbia ${ }^{31}$ require day or hour limits for prescription drugs dispensed in the context of an emergency. Emergency-basis dispensing in this context encompasses a

\footnotetext{
${ }^{25}$ See, e.g., Ala. Admin. Code r. 560-X-16-.28 (eff. date unclear); ARK. Admin. Code 016.06.22-213.100; CAL. COdE Regs. tit. 22, § 51313 (eff. date unclear); Colo. CODE REgS 2505-10:8.800 (eff. date unclear, 2009 or 2012); CONN. GEN. StAT. ANN. § 17b-494 (eff. date unclear); 16 DEL. C. § 3005B (eff. 2000); FLA. STAT. ANN. § 409.912 (eff. date unclear); HAW. CODE R. § 17-1739.1-4.1 (eff. date unclear); ILL. AdMIN. CODE tit. 86, § 530.150 (eff. date unclear); Ky. REV. STAT. ANN. § pt. I (eff. date unclear); 10-144-101 Me. Code R. § 80 (eff. 2005); MD. Code Regs. 10.09.03.05 (eff. 2003 ); 130 MASS. Code Regs. 406.411 (eff. date unclear); Mich. CoMp. LAWs AnN. § 333.9709 (eff. 2004); Minn. StAT. ANN. $\S 256 B .0625$ (eff. 2010); 23-214 MISS. CODE R. § 1.6 (eff. 2012); Mo. CodE REGS. ANN. tit. 13, § 70-20.045 (eff. date unclear, 2000-2001); Mont. AdMIN. R. 37.86.1102 (eff. date unclear); NEB. AdMIN . R. \& Regs. tit. 471, ch. $16 \S 004$ (eff. date unclear); N.H. COdE AdMIN. R. HE-W 570.06 (eff. date unclear); N.J. STAT. ANN. § 30:4D-22 (eff. 1975 ); N.M. CODE R. § 8.324.4 (eff. date unclear); N.Y. COMP. CODES R. \& REGS. tit. 18, § 505.3 (eff. date unclear); 10A N.C. ADMIN. CODE 25K.0201 (eff. date unclear); N.D. AdMIN. CODE 75-02-02-27 (eff. date unclear); OHIO ADMIN. CODE 5101:3-9-03 (eff. date unclear); OKLA. AdMIN. CODE § 317:30-5-77.1 (eff. 2000); Or. ADMIN. R. 410-121-0060 (eff. date unclear); 72 PA. StAT. ANN. § 3761-511 (eff. 1996); 22-1-3 R.I. CODE R. §III (eff. date unclear); TENN. CoMP. R. \& REgS. 1200-13-13.04 (eff. date unclear); TEX. Hum. RES. CODE ANN. §32.024 (eff. 2003); UTAH ADMIN. CODE r. 414-60B (eff. 2009 ); 12 VA. ADMIN. CODE § 30-50-210 (eff. date unclear); WIS. ADMIN. CODE DHS § 109.31 (eff. date unclear, 2003 or 2008 ); 10 WYO. CODE R. HEALTH $\S 10$ (eff. 2005).

${ }^{26}$ D.C. CODE MUN. ReGs. tit. 29, § 2705 (eff. 2012).

27 OKLA. ADMIN. CODE § 317:30-5-77.1 (eff. 2000).

${ }^{28}$ ILL. ADMIN. CODE tit. 86, §530.150 (eff. date unclear).

${ }^{29} 10$ WYO. CODE R. HEALTH § 10(b)(3) (eff. 2005).

${ }^{30}$ See, e.g., ALA. CODE § 34-23-75 (eff. 1991); ARK. CODE ANN. § 17-92-102 (eff. date unclear); ColO. REV. STAT. ANN. § 18-18-414 (eff. 1992); CONN. GEN. STAT. ANN. § 17b-491a (eff. date unclear); FLA. STAT. ANN. § 465.0275 (eff. date unclear); HAW. CODE R. § 17-1739.1-4.1 (eff. date unclear); KAN. STAT. ANN. § 65-1637 (eff. 2005); 907 KY. ADMIN. REGS. 17:005 (eff. 2012); 10-144-101 ME. CODE R. § 80 (eff. 2005); MD. CODE REGS. 10.09.03.06 (eff. date unclear); MICH. COMP. LAWS ANN. § 333.9709 (eff. 2004); 30-20 MISS. CODE R. § 3001:XIX (eff. 2012); 471 NEB. AdMIN. CODE § 16-004 (eff. date unclear); N.H. COdE AdMIN. R. Ph 704.15 (eff. date unclear); N.J. AdMIN. COdE § 10:51-1.14 (eff. date
} 
range of situations. ${ }^{32}$ This includes situations wherein a pharmacist may dispense a limited supply of a drug for a patient with an immediate medical need and the prescriber cannot be reached. Another emergency situation is when a pharmacist may dispense a limited quantity of a drug when there is a medical need but prior authorization has not been obtained from the patient's benefit plan. For example, New Mexico law states

The Pharmacy Act does not prohibit: . . (9) a pharmacist from exercising his professional judgment in refilling a prescription for a prescription drug, unless prohibited by another state or federal law, without the authorization of the prescribing licensed practitioner, if: (a) failure to refill the prescription might result in an interruption of a therapeutic regimen or create patient suffering; (b) the pharmacist is unable to contact the licensed practitioner after reasonable effort; (c) the quantity of prescription drug dispensed does not exceed a seventy-two hour supply; (d) the pharmacist informs the patient or the patient's agent at the time of dispensing that the refill is being provided without such authorization and that authorization of the licensed practitioner is required for future refills; and (e) the pharmacist informs the licensed practitioner of the emergency refill at the earliest reasonable time. ${ }^{33}$

Eleven states ${ }^{34}$ allow for emergency-basis dispensing but specifically exclude Schedule II drugs. For example, an Alabama statute on emergency prescription refills provides:

In the event a pharmacist receives a request for a prescription refill and the pharmacist is unable to readily obtain refill authorization from the prescriber, the pharmacist may dispense a onetime emergency refill of up to a [seventy-two] hour supply of the prescribed medication, providing that: (1) The prescription is not a medicinal agent listed in Schedule II . . . (2) The medication is essential to the maintenance of life or the continuation of therapy in a chronic condition ... ${ }^{35}$

unclear); N.M. StAT. ANN. § 61-11-7 (eff. date unclear); N.Y. COMP. CODES R. \& REGS. tit. 10, § 80.63 (eff. date unclear); 10A N.C. AdMIN. CODE 25K.0201 (eff. date unclear); N.D. AdMIN. CODE 75-02-02-27 (eff. date unclear); OHIO AdMIN. CODE 5101:3-9-03 (eff. date unclear); OKLA. STAT. ANN. tit. 63, § 5030.5 (eff. date unclear); OR. ADMIN. R. 410121-0060 (eff. date unclear); R.I. GEN. LAWS ANN. § 5-19.1-24 (eff. 2001); TENN. CODE ANN. § 63-10-207 (eff. date unclear, 1997 or 2007); TEX. Occ. CODE ANN. § 562.054 (eff. date unclear, 1999 or 2007); UTAH ADMIN. CODE r. 15617b (eff. date unclear); 12 VA. ADMIN. CODE § 30-50-210 (eff. date unclear); WASH. ADMIN. CODE. § 246-869-100 (eff. date unclear).

${ }^{31} 29$ D.C. CODE MUN. REGS. § 2705 (eff. 2012).

${ }^{32}$ Laws pertaining to declared states of emergency are not included in the scope of research conducted.

${ }^{33}$ N.M. STAT. ANN. § 61-11-7 (eff. date unclear).

${ }^{34}$ See, e.g., ALA. CODE § 34-23-75 (eff. 1991); ARK. CODE ANN. § 17-92-102 (eff. date unclear); FLA. STAT. ANN. § 893.04 (eff. 2009); KAN. Stat. ANN. § 65-1637 (eff. 2005); N.H. COdE AdMIN, R. Ph 704.15 (eff. date unclear); 21 N.C. AdMIN. CODE 46.1809 (eff. date unclear); N.D. CENT. CODE ANN. 43-15-01 (eff. date unclear); OHIO REV. CODE ANN. § 4729.281 (eff. 1998); R.I. GeN. LAWS ANN. § 5-19.1-24 (eff. 2001); TEX. Occ. CODE ANN. § 562.054 (eff. date unclear, 1999 or 2007); UTAH ADMIN. CODE r. 156-17b (eff. date unclear).

${ }^{35}$ ALA. CODE $\S 34-23-75$ (eff. 1991). 
Twenty-two states ${ }^{36}$ permit emergency dispensing for members of benefit plans when a medical need is determined but required prior authorization has not been obtained. Kentucky regulation reads as follows

(4) If a prescription for an enrollee is for a non-preferred drug and the pharmacist cannot reach the enrollee's primary care provider or the MCO for approval and the pharmacist determines it necessary to provide the prescribed drug, the pharmacist shall: (a) Provide a seventy-two (72) hour supply of the prescribed drug; or (b) Provide less than a seventy-two (72) hour supply of the prescribed drug, if the request is for less than a seventy-two (72) hour supply . . ${ }^{37}$

Nebraska law regarding its Medical Assistance Program also provides that

The NE-Pop or the Department will respond to any request for prior authorization within 24 hours of receipt of the request. In cases of medical emergency, provisions are made for dispensing a seventy-two (72) hour supply of a covered outpatient prescribed medication. ${ }^{38}$

Similarly, Oregon's Medical Assistance program law has a section stating

Emergency need: The Pharmacist may request an emergent or urgent dispensing from the Pharmacy Benefits Manager (PBM) when the client is eligible for covered fee-for-service drug prescriptions. (a) Clients who do not have a [prior authorization] pending may receive an emergency dispensing for a 96-hour supply. (b) Clients who do have a [prior authorization] pending may receive an emergency dispensing up to a seven-day supply. ${ }^{39}$

\section{Prescription Drug Refills}

Twenty states' ${ }^{40}$ laws set time limits for prescription drug refills. For example, California law provides that prescriptions for Schedule III or IV drugs may not exceed a total of a 120-day supply across a

${ }^{36}$ See, e.g., ALA. AdMIN. CODE r. 560-X-16-.28 (eff. date unclear); ARK. AdMIN. CODE 016.06.22-240.000 (eff. 2005 ); Colo. COde Regs 2505-10:8.800 (eff. date unclear, 2009 or 2012); ConN. GEn. STAT. ANN. § 17b-491a (eff. date unclear); FLA. StAT. ANN. § 409.912 (eff. date unclear); HAW. CODE R. § 17-1739.1-4.1 (eff. date unclear); 907 KY. AdMin. Regs. 17:005 (eff. 2012); 10-144-101 Me. Code R. § 80 (eff. 2005); MD. Code Regs. 10.09.03.06 (eff. date unclear); Mich. COMP. LAWS ANN. § 333.9709 (eff. 2004); 23-214 Miss. Code R. § 1.10 (eff. 2012); NEB. AdMIN. R. \& ReGs. tit. 471, ch. $16 \S 004$ (eff. date unclear); N.H. CODE AdMIN. R. HE-W 570.06 (eff. date unclear); N.J. ADMIN. CODE $\S 10: 51-1.14$ (eff. date unclear); N.D. ADMIN. CODE 75-02-02-27 (eff. date unclear); OHIO ADMIN. CODE 5101:3-9-03 (eff. date unclear); OKLA. ST. ANN. tit. 63, § 5030.5 (eff. date unclear); Or. ADMIN. R. 410-121-0060 (eff. date unclear); TENN. COMP. R. \& REgs. 1200-13-13-.11 (eff. date unclear); TEX. Gov'T CODE ANN. § 531.073 (eff. 2003); UTAH AdMIN. CODE r. 414-60B (eff. 2009); 12 VA. ADMIN. CODE $§ 30-50-210$ (eff. date unclear).

37907 KY. ADMIN. REGS. 17:005 (eff. 2012).

${ }^{38}$ NEB. AdMIN . R. \& REGS. tit. 471, ch. $16 \S 004$ (eff. date unclear).

39 OR. AdMIN. R. 410-121-0060 (eff. date unclear).

${ }^{40}$ See, e.g., Ala. CODE § 34-23-75 (eff. 1991); Ark. Code AnN. § 17-92-102 (eff. date unclear); WeSt's ANN. CAL. HEALth \& SAFETY CODE $\S 11200$ (eff. 1992); Colo. COde Regs 2505-10:8.800 (eff. date unclear, 2009 or 2012); FLA. STAT. ANN. $\S 465.0275$ (eff. date unclear); HAW. REV. STAT. § 328-16 (eff. date unclear); ILL. AdMIN. CODE tit. 86, § 530.150 (eff. date unclear); KAN. STAT. ANN. § 65-1637 (eff. 2005); KY. REV. STAT. § 217.215 (eff. date unclear); N.H. CODE ADMIN., R. Ph 704.15 (eff. date unclear); N.J. AdMIN. CODE § 10:51-1.15 (eff. 1998); N.M. STAT. ANN. § 61-11-7 (eff. date unclear); 21 N.C. AdMIN. CODE 46.1809 (eff. date unclear); N.D. CENT. CODE ANN. 43-15-01 (eff. date unclear); OHIO REV. CODE ANN. § 4729.281 (eff. 1998); R.I. GEN. LAWS ANN. § 5-19.1-24 (eff. 2001); TENN. CoMP. R. \& REgS. 1200-13-13-.04 (eff. 
specified number of refills. Washington law on prescription record requirements includes the following language

(f) Emergency refills - If the prescriber is not available and in the professional judgment of the pharmacist an emergency need for the medication has been demonstrated, the pharmacist may dispense enough medication to last until a prescriber can be contacted - but not to exceed 72 hours' supply. The prescriber shall be promptly notified of the emergency refill. ${ }^{41}$

\section{Pain Management Clinic Setting}

Two states ${ }^{42}$ have set prescription drug limits for pain management clinics. Louisiana law on pain management clinic operations provides that

A clinic shall establish practice standards to assure quality of care, including but not limited to, requiring that a prescription for a controlled dangerous substance may have a maximum quantity of a [thirty] day supply and shall not be refillable. ${ }^{43}$

West Virginia statute stipulates that "[a] pain management clinic shall not dispense to any patient more than a seventy-two-hour supply of a controlled substance." 44

\section{Dosage Limitations}

Another category of prescription drug limit laws limit the dosage amounts of prescription drugs.

\section{Dosage Limits Applicable to Certain Drugs or Types of Prescriptions}

Some states ${ }^{45}$ and the District of Columbia ${ }^{46}$ set dosage limits based on the type of drug schedule.

Rhode Island law describes a "dosage unit" as a "single capsule, tablet or suppository, or not more than one five ... [milliliters] of an oral liquid" and states that "[p]rescriptions in Schedule III cannot be written for more than [100] dosage units and not more than [100] dosage units may be dispensed at one time."

date unclear); TEX. Occ. CODE ANN. § 562.054 (eff. date unclear, 1999 or 2007); UTAH ADMIN. CODE r. 156-17b (eff. date unclear); WASH. ADMIN. CODE. § 246-869-100 (eff. date unclear). ${ }^{41}$ WASH. ADMIN. CODE. 246-869-100 (eff. date unclear).

${ }^{41}$ WASH. ADMIN. CODE. 246-869-100 (eff. date unclear).

${ }^{42}$ Louisiana and West Virginia.

${ }^{43} 48$ LA. ADMIN. CODE $\S 7833$ (eff. 2008).

${ }^{44}$ W. VA. CODE $§ 16-5 \mathrm{H}-4$ (eff. 2012).

${ }^{45}$ Fifteen states. See, e.g., ARIz. Rev. StAt. ANn. §36-2525 (eff. date unclear); ARK. AdMIN. CODE 070.00.7-07-04-0007 (eff. 2011); 24 Del. AdMIN. CODE $§ 4.0$ (eff. 2009); GA. COMP. R. \& REGS. 480-19-.01 (eff. 2011); KAN. AdMIN. REGS. § 6820-22 (eff. 1978); 902 Ky. ADMIN. REGS. 55:035 (eff. date unclear); LA. AdMIN. CODE tit. 46, pt. LIII, § 2747 (eff. 2008); Mo. CODE REGS. ANN. tit. 19, § 30-1.074 (eff. date unclear); N.J. AdMIN. CODE § 13:45H-7.19 (eff. date unclear); N.M. CODE R. § 16.19.20 (eff. 2002); N.Y. COMP. CODES R. \& ReGs. tit. 10, § 80 (eff. date unclear); OHIO AdMin. CODE 472911-09 (eff. date unclear); R.I. GEN. LAWS ANN. § 21-28-3.18 (eff. 2006); 22 TEX. AdMIN. CODE § 291.33 (eff. date unclear); W. VA. CODE R. § 15-2-7 (eff. date unclear).

${ }^{46}$ D.C. MUN. REGS. tit. 22-B, § 1314 (eff. 1986). 
For Schedule IV and V drugs, the law states that prescriptions "may be written for up to a ninety [90] day supply based on directions. No more than [360] dosage units may be dispensed at one time." ${ }^{47}$

Arkansas regulation provides the following:

A controlled substance listed in Schedule V ... may be dispensed by a pharmacist without a prescription to a purchaser at retail, provided that: . . (b) Not more than $240 \mathrm{cc}$. (8 ounces) of any such controlled substance containing opium, ... nor more than 48 dosage units of any such controlled substance containing opium, nor more than 24 dosage units of any other such controlled substance may be dispensed at retail to the same purchaser in any given 48 -hour period... ${ }^{48}$

\section{Dosage Limits Applicable to Certain Benefit Plans}

Six states ${ }^{49}$ set dosage limits for members of certain benefit plans. For example, Delaware's Prescription Drug Payment Assistance Program provides that "[n]o drug prescription may exceed 100 dosing units or a [thirty-four]-day supply, whichever is greater." ${ }^{50}$ Similarly, New Jersey's statute on its Pharmaceutical Assistance to the Aged and Disabled Program states that "[t]he commissioner may allow up to a [sixty] day supply or 100 unit doses, whichever is greater, of prescription drugs used in the treatment of chronic maintenance conditions." ${ }^{51}$

\section{Conclusion}

This inventory provides a collection of laws on prescription drug time or dosage limits. Additional legal requirements or penalties related to prescription drug overdose fall outside the scope of this section. This inventory does not contain a full assessment of all relevant prescription drug laws, which may include provisions setting forth professional licensing penalties or criminal sanctions. Practitioners should consult with legal counsel to become fully informed of the legal landscape concerning prescription drugs and how the laws are implemented and enforced in their state.

This document was written by researchers in the Public Health Law Program in the Office for State, Tribal, Local and Territorial Support, ${ }^{52}$ with assistance from the Division of Unintentional Injury Prevention in the National Center for Injury Prevention and Control. ${ }^{53}$ For further technical assistance

\footnotetext{
${ }^{47}$ R.I. GEN. LAWS § 21-28-3.18(eff. 2006).

${ }^{48}$ ARK. AdMIN. CODE 070.00.7-07-04-0007 (eff. 2011).

${ }^{49}$ See, e.g., Conn. Gen. StAT. AnN. § 17b-494 (eff. date unclear); 16 Del. C. § 3005B (eff. 2000); N.J. STAT. ANN. $\S 30: 4 D-22$ (eff. 1975); N.Y. COMP. CODES R. \& REGS. tit. 18, § 505.3 (eff. date unclear); 72 PA. STAT. ANN. § $3761-511$ (eff. 1996); 22-1-3 R.I. CODE R. § X (eff. date unclear).

${ }^{50}$ DEL. CODE ANN. tit. 16, § 3005B (eff. 2000).

${ }^{51}$ N.J. STAT. ANN. § 30:4D-22 (eff. 1975).

${ }^{52}$ Akshara Menon, JD, MPH, Carla Chen, JD, and Matthew Penn, JD, MLIS.

${ }^{53}$ Noah Aleshire, JD, and Leonard Paulozzi, MD, MPH.
} 
with this inventory or prescription drug laws, please contact the Public Health Law Program. ${ }^{54}$ For technical assistance on all other opioid pain reliever-related topics, please contact the Division of Unintentional Injury Prevention. ${ }^{55}$

PHLP provides technical assistance and public health law resources to advance the use of law as a public health tool. PHLP cannot provide legal advice on any issue and cannot represent any individual or entity in any matter. PHLP recommends seeking the advice of an attorney or other qualified professional with questions regarding the application of law to a specific circumstance. The findings and conclusions in this summary are those of the author and do not necessarily represent the official views of the Centers for Disease Control and Prevention.

This menu includes laws enacted through September 28, 2012.

Published March 5, 2015.

\footnotetext{
${ }^{54}$ Public Health Law Program, Centers for Disease Control and Prevention, 4770 Buford Hwy. NE, M.S. E-70, Atlanta, GA 30341. Telephone: (404) 498-0470. Fax: (404) 498-6882. Email: MPenn@cdc.gov. Web: http://www.cdc.gov/phlp.

${ }^{55}$ Division of Unintentional Injury Prevention, National Center for Injury Prevention and Control, Centers for Disease Control and Prevention, 4770 Buford Hwy. NE, M.S. F-62, Atlanta, GA 30341. Email: LPaulozzi@cdc.gov. Web: http://www.cdc.gov/HomeandRecreationalSafety/Poisoning/laws/index.html.
} 